\title{
La coevaluación como estrategia para mejorar la dinámica del trabajo en grupo: una experiencia en Ciencias del Deporte
}

\section{Co-evaluation as a strategy to improve working group dynamics: an experience in Sport Sciences}

\author{
Maribel BARRIOPEDRO MORO, Cristina LÓPEZ DE SUBIJANA, Miguel Ángel \\ GÓMEZ RUANO y Antonio RIVERO HERRAIZ \\ Universidad Politécnica de Madrid
}

Recibido: Octubre 2014

Evaluado: Enero 2015

Aceptado: Febrero 2015

\section{Resumen}

El objetivo del presente estudio fue evaluar si las percepciones de los alumnos antes y después de la realización del trabajo, es decir, su percepción sobre diversos aspectos del funcionamiento del grupo y las competencias de trabajo adquiridas y las que consideran que deben mejorar, difería en función de que la contribución de los distintos miembros del grupo fuese o no coevaluada. Participaron 144 alumnos de Ciencias de la Actividad Física y el Deporte. Para analizar la percepción de los alumnos del trabajo en grupo se utilizó el cuestionario adaptado por Bourne et al. (2001). Los resultados mostraron que los grupos en los se introdujo la coevaluación valoraron de forma más negativa la experiencia en general que los grupos en los que no se introdujo la coevaluación. Sin embargo, los grupos con coevaluación percibieron que su competencia para trabajar en equipo había mejorado en mayor medida que los grupos sin coevaluación, valorando de forma más positiva tanto el funcionamiento como el resultado del trabajo e incrementaron sus conocimientos sobre el resto de los componentes. El uso de un sistema de coevaluación como el propuesto parece generar una mejor valoración tanto del funcionamiento del equipo como del resultado de su trabajo.

Palabas clave: trabajo en grupo; coevaluación, educación universitaria;

\begin{abstract}
The aim of this study was to analyze if the perceptions of students before and after carrying out the work, that is, their perception of different aspects of the functioning of the group, the working skills acquired as well as those they think that need to be improved, varied depending on whether the contribution of the different members of the group was being co-evaluated or not. 144 students of Physical Activity and Sport Sciences participated in this study. In order to analyze the students' perception of group work the adapted questionnaire by Bourne et al. (2001) was used. Results showed that groups which implemented co-evaluation assessed more negatively the experience in general than those which did not. However, co-evaluation groups


perceived their competence to work as a team had improved to a greater extent than the groups without co-evaluation, evaluating more positively both the performance and the result of work and increasing their knowledge of the other team members. Using a co-evaluation system seems to generate both a better assessment of the running of the team and the result of its work.

Keywords: group work; co-evaluation; higher education.

La introducción de competencias como objetivo prioritario de la formación universitaria hace necesaria la utilización de metodologías activas, centradas en la capacitación en competencias propias de la disciplina. Dentro de las metodologías activas que se pueden emplear en el Espacio Europeo de Educación Superior (EEES) se encuentran el aprendizaje colaborativo en grupo, el aprendizaje orientado a proyectos y problemas, la resolución de casos o el portfolio. Sin embargo, una de las estrategias didácticas más empleadas en el aula universitaria es el trabajo en grupo (Alías, Gil, Riscos, Valcárcel y Vicario, 2006).

El trabajo en grupos es una estrategia didáctica que ha sido empleada y analizada en las aulas desde los años 70 en el ámbito de la psicología y la sociología del aprendizaje. De acuerdo con los autores especializados el trabajo en grupos ha desarrollado los planteamientos constructivistas como mejora de los procesos de enseñanza- aprendizaje, y además supone una estrategia esencial para implicar a los estudiantes en la reconstrucción de conocimientos. El trabajo en grupo es una estrategia que se ha modificado con el paso de los años, en la actualidad este modelo ha evolucionado hacia el aprendizaje cooperativo (Santos-Rego, Lorenzo-Moledo, y Priegue-Caamaño, 2009; Vilches y Gil-Pérez, 2011).

El trabajo en grupo ha sido establecido como una estrategia básica para la adquisición de competencias inter-personales en el EEES. En particular, su uso potencia y favorece las destrezas sociales, la cooperación, la interacción y el trabajo en equipo (Gámez y Torres, 2013). La importancia del trabajo en grupo radica en su utilización dentro del futuro laboral y profesional de los estudiantes (Vallejo y Molina, 2011). En este sentido el trabajo en grupo no debe considerarse como una mera agrupación de estudiantes, sino que implica trabajar juntos y colaborar en una misma meta con objetivos claramente identificados (Moreno, Bajo, Moya, Maldonado y Tudela, 2007). Asimismo, el trabajo en "grupo" se denomina así debido a las decisiones que han de tomar los miembros del mismo (i.e., tomar decisiones, resolver problemas, establecer conclusiones, etc.). De este modo el grupo supone más que la suma individual de cada alumno, primando los objetivos del grupo (Moreno et al., 2007).

Dentro del aula universitaria el uso de dinámicas de grupo permite la aplicación de las estrategias de trabajo en grupo, este planteamiento ha sido ampliamente descrito dentro del EEES (Blanco, 2009; Colas y De Pablos, 2005; Gámez y Torres, 2013; López, 2005; Moreno et al., 2007).

No obstante, el trabajo en grupo no está libre de dificultades tanto para el profesor como para los estudiantes (Burdett, 2007). A menudo, es tipo de trabajo da lugar a ambientes de trabajos tensos o cargados emocionalmente, puesto que implica mucho 
más que la mera habilidad del estudiante en la materia en cuestión (Yazici, 2005; Johnson y Johnson, 1994). El trabajo en grupo requiere que los estudiantes entiendan los procesos grupales, sean capaces de coordinar tareas y manejar las relaciones interpersonales, así como desarrollar ciertas habilidades para la resolución de conflictos (Furnham, 1997). Estas demandas del trabajo grupal hacen que en muchas ocasiones los alumnos se muestren poco entusiasmados (McConnell, 2005). Así, por ejemplo, Livingstone y Lynch (2002) encuentran que los estudiantes informan sentirse "estafados" con el trabajo en grupo y Crebert, Bates, Bell, Patrick y Cragnolini (2004) encuentran que los alumnos se muestran descontentos con la supervisión del proceso y prefieren trabajar de forma individual. Estos autores sugieren que para conseguir los beneficios asociados al trabajo en grupo, los docentes no pueden desligarse de los aspectos problemáticos que surgen en una dinámica grupal y deben utilizar estrategias que faciliten dicho proceso. Burdett (2007) encuentra que las principales dificultades que aparecen en la dinámica grupal están relacionadas con la falta de asistencia a las reuniones del grupo, el exceso de liderazgo por parte del algún miembro, la intromisión de los miembros en las tareas correspondientes a otro estudiante y la falta de contribución al trabajo por parte de alguno de ellos. Cuando las habilidades y los esfuerzos de otros afectan a la recompensa que obtiene un individuo a través de la calificación del trabajo, estas disfuncionalidades del grupo cobran aún una mayor importancia.

Muchos expertos en el ámbito educativo consideran que el trabajo en grupo funciona mejor si la calificación del trabajo grupal se ajusta al rendimiento de cada participante (Kaufman, Felder y Fuller, 2000). Si este ajuste no se realiza, aquellos alumnos que no hayan contribuido al resultado recibirían la misma calificación que aquellos que si lo hicieron. Draper (2007) señala que la autoevaluación y la coevaluación en el trabajo en equipo incrementan la autonomía de los estudiantes y el feedback que obtienen de su proceso de aprendizaje. La evaluación entre iguales, además, contribuye a mejorar la participación de los alumnos y a desarrollar habilidades tales como la negociación, la diplomacia, aprender a dar y recibir críticas, a cómo justificar la posición adoptada, etc. (Liu, Lin, Chiu y Yuan, 2001). Johnston y Miles (2004) encuentran que los estudiantes pueden juzgar de forma bastante precisa las contribuciones del resto de los miembros del grupo, sobre todo si se les pide una evaluación holística que refleje la contribución de cada miembro (Lejk y Wyvill, 2001).

En el presente estudio se implementó la coevaluación como instrumento para mejorar la comunicación y la dinámica del grupo. Por lo tanto, el objetivo del presente estudio fue evaluar si las percepciones de los alumnos antes y después de la realización del trabajo, es decir, su percepción sobre diversos aspectos del funcionamiento del grupo y las competencias de trabajo adquiridas y las que consideran que deben mejorar, difería en función de que la contribución de los distintos miembros del grupo fuese o no coevaluada. 


\section{Método}

\section{Muestra}

Participaron 144 estudiantes (114 hombres y 30 mujeres) de $1^{\circ}$ (20,4 $\pm 2,3$ años) de $\mathrm{CC}$ de la AF y el Deporte de la Universidad Politécnica de Madrid que cursaban la asignatura de Estadística y TIC's. La asignatura de Estadística se imparte a cuatro grupos de 68, 72, 71 y 69 alumnos cada uno. En dos de los grupos seleccionados al azar, se implantó la coevaluacion de la participación en el trabajo, mientras que en otros dos grupos no se utilizó dicho sistema. Al finalizar la asignatura, 76 participaron en grupos de trabajo en los que debían evaluar la contribución al mismo de los diferentes miembros, y 68 participaron en grupos en los que no se realizaba dicha evaluación. La cumplimentación del cuestionario fue voluntaria.

\section{Instrumentos}

Para analizar la percepción que los alumnos tenían del trabajo en grupo se utilizó el cuestionario de Falchicov (1988) y de Garvin et al. (1995) adaptado por Bourner, Hughes y Bourner (2001). En este cuestionario se evalúan las percepciones de los alumnos antes y después de la realización del trabajo, su percepción sobre diversos aspectos del funcionamiento del grupo y las competencias de trabajo que perciben que han adquirido y las que deben mejorar.

\section{Procedimiento}

La asignatura se podía cursar por evaluación continua o por evaluación final. En el caso de los alumnos que optaban por evaluación continua, dos exámenes parciales suponían el $85 \%$ de la nota, mientras que los informes sobre los que se realizaba la coevaluacion, un $15 \%$ de la nota final. Los alumnos de evaluación continua debían entregar 3 informes acerca de la realización del apartado de resultados de estudios realizados en el ámbito profesional. Cada informe de coevaluacion tenía un peso del 5 $\%$ de la nota. Los informes requeridos implicaban aplicar tres bloques de contenidos temáticos: estadística descriptiva univariada, estadística descriptiva bivariada y estadística inferencial. Los grupos estaban constituidos por 3 alumnos que se eligieron entre ellos. El trabajo debían desarrollarlo a lo largo de 10 semanas. Los grupos que valoraban la contribución de cada miembro al trabajo lo hicieron en tres ocasiones (en la $2^{\mathrm{a}}, 5^{\mathrm{a}}$ y $8^{\mathrm{a}}$ semana) utilizando para ello una escala de 6 puntos reflejando los diferentes niveles de participación (Morales, 2008) (0: ausente; 1: nivel mínimo; 2: por debajo de la media; 3: normal, promedio; 4: por encima de la media del grupo y 5: Excelente, sobresale de manera apreciable). Para facilitar la evaluación se les proporcionaron criterios para cada uno de los niveles (0: No participó en absoluto; 1 : Faltó muchas veces a las reuniones o no completó su tarea, rara vez estaba preparado; 2: Habitualmente hizo los que se supone que tenía que hacer, preparación y colaboración mínimas; 3: Habitualmente hizo lo que se supone que tenía que hacer, preparación y colaboración aceptables; 4 . Hizo su tarea de forma consistente, buena preparación, cooperador; 5: Ayuda mucho a los demás, hace más de lo que le 
corresponde). Un promedio, en la valoración de un sujeto por parte de sus compañeros, inferior a 3 suponía obtener una calificación igual a cero en el trabajo. Esta información fue suministrada a los dos grupos que realizaban coevaluación en la presentación de la asignatura, al comienzo del semestre. El cuestionario acerca de sus percepciones del trabajo en grupo de Falchicov (1988) y de Garvin et al. (1995) adaptado por Bourner, Hughes y Bourner (2001) fue administrado a través de la plataforma de la asignatura, al finalizar el cuatrimestre en mayo de 2013. El cuestionario era anónimo y su cumplimentación voluntaria.

\section{Análisis estadístico}

Para el análisis de los resultados se utilizó el programa SPSS versión18. Para analizar las diferencias en función de la coevaluación para las variables nominales se utilizó la prueba Chi Cuadrado de Pearson y para analizar estas diferencias en los conjuntos de variables ordinales que suponían la valoración de un único aspecto (valoración de la experiencia, de sus sensaciones, de los aspectos mejorados) se aplicó un análisis multivariado de varianza de un factor (MANOVA) tras comprobar el supuesto de igualdad de varianzas o de dos factores para evaluar las diferencias antes y después de realizar el trabajo en función de la coevaluación. El criterio para evaluar las diferencias multivariadas fue la Traza de Pillai. Por último, la prueba U de MannWhitney fue aplicada para comparar los grupos en variables ordinales y la t de Student para comparar las calificaciones obtenidas en el trabajo. El nivel de riesgo se fijó en 0,05 .

\section{Resultados}

En la Tabla 1 se presentan las valoraciones de la experiencia de trabajar en equipo en función de que la contribución al trabajo fuese o no coevaluada. La introducción o no de coevaluación en los grupos tuvo un efecto principal significativo sobre la valoración de la experiencia de trabajar en grupo $\left(\mathrm{F}_{7,136}=3,84 ; \mathrm{p}<0,01\right)$. Los contrastes univariados pusieron de manifiesto que los sujetos que participaron en equipos sin coevaluación valoraron la experiencia de trabajar en equipo como más agradable $\left(\mathrm{F}_{1,142}=18,3 ; \mathrm{p}<0,01\right)$, mas inspiradora $\left(\mathrm{F}_{1,142}=15,5 ; \mathrm{p}<0,01\right)$, como una experiencia de aprendizaje más positiva $\left(\mathrm{F}_{1,142}=5,7 ; \mathrm{p}<0,05\right)$ y más creativa $\left(\mathrm{F}_{1,142}=\right.$ $15 ; \mathrm{p}<0,01)$ que aquellos que participaron en grupos con coevaluación. No se observaron diferencias significativas en el grado de dificultad con la que se valoró la experiencia $\left(\mathrm{F}_{1,142}=1,5 ; \mathrm{p}>0,05\right)$, ni en los satisfactoria $\left(\mathrm{F}_{1,142}=3,7 ; \mathrm{p}>0,05\right)$ y beneficiosa $\left(\mathrm{F}_{1,142}=3,7 ; \mathrm{p}>0,05\right)$ que resultó en función de la introducción o no de la coevaluación. 


\begin{tabular}{llcc}
\hline Escala de 1 a 5 & Agradable & $\begin{array}{c}\text { Con Coevaluación } \\
(\mathbf{N = 7 6 )}\end{array}$ & $\begin{array}{c}\text { Sin Coevaluación } \\
(\mathbf{N = 6 8})\end{array}$ \\
\hline No Agradable & Inspiradora & $2,39 \pm 0,9$ & $4,03 \pm 0,9 * *$ \\
\hline No Inspiradora & Fácil & $3,11 \pm 1,0$ & $3,51 \pm 1,0^{* *}$ \\
\hline Difícil & Satisfactoria & $3,28 \pm 1,0$ & $3,31 \pm 1,0$ \\
\hline Frustrante & Beneficiosa & $3,46 \pm 0,9$ & $3,78 \pm 1,1$ \\
\hline Nada beneficioso & De aprendizaje & $3,54 \pm 0,9$ & $3,90 \pm 1,0 *$ \\
\hline De aprendizaje negativa & positiva & $2,91 \pm 1,0$ & $3,59 \pm 1,2 * *$ \\
\hline Algo no creativo & Algo muy creativo & & \\
\hline$* 0<0,05 * * \mathrm{p}<0,01$ & & &
\end{tabular}

Tabla 1. Valoraciones sobre la experiencia de trabajar en equipo (Media \pm Desviación típica)

En la Tabla 2 se presentan las percepciones de los alumnos sobre cómo se sentían al comenzar y al terminar el trabajo en equipo en función de la introducción o no de la coevaluación. La introducción o no de coevaluación en los grupos tuvo un efecto principal significativo sobre la percepción de los alumnos de trabajar en grupo $\left(\mathrm{F}_{7,136}=\right.$ $3,32 ; \mathrm{p}<0,01)$. Los contrastes univariados pusieron de manifiesto que los sujetos que participaron en equipos sin coevaluación percibieron la experiencia de trabajar en equipo con más entusiasmo $\left(\mathrm{F}_{1,142}=7,1 ; \mathrm{p}<0,01\right)$ y se percibieron más creativos $\left(\mathrm{F}_{1,142}\right.$ $=10,7 ; \mathrm{p}<0,01)$. En el resto de las dimensiones evaluadas no se encontraron diferencias significativas $\left(\mathrm{F}_{1,142}<1\right.$ para las 5 dimensiones $)$. El efecto multivariado del momento resulto significativo $\left(\mathrm{F}_{7,136}=19,2 ; \mathrm{p}<0,01\right)$ pero no su interacción con la introducción o no de la coevaluación $\left(\mathrm{F}_{7,136}=1,2 ; \mathrm{p}>0,05\right)$. Tanto los alumnos que participaron en equipos con coevaluación como aquellos que participaron en equipos sin coevaluación tras la realización del trabajo en equipo se percibieron con más conocimientos de lo estudiado $\left(\mathrm{F}_{1,142}=113,3 ; \mathrm{p}<0,01\right)$, con más confianza $\left(\mathrm{F}_{1,142}=\right.$ $41,1 ; \mathrm{p}<0,01)$, más flexibles en sus opiniones $\left(\mathrm{F}_{1,142}=22,5 ; \mathrm{p}<0,01\right)$, mas independientes $\left(\mathrm{F}_{1,142}=9,4 ; \mathrm{p}<0,01\right)$, más competentes $\left(\mathrm{F}_{1,142}=38,1 ; \mathrm{p}<0,01\right)$ y más creativos $\left(\mathrm{F}_{1,142}=25,6 ; \mathrm{p}<0,01\right)$. Sin embargo, al finalizar el trabajo no se percibieron mas entusiasmados que al comenzarlo $\left(\mathrm{F}_{1,142}=1,3 ; \mathrm{p}>0,05\right)$. 


\begin{tabular}{|c|c|c|c|c|c|}
\hline \multirow[t]{2}{*}{ Escala de 1 a 5} & & \multicolumn{2}{|c|}{$\begin{array}{c}\text { Con } \\
\text { Coevaluación } \\
(\mathrm{N}=76) \\
\end{array}$} & \multicolumn{2}{|c|}{$\begin{array}{c}\text { Sin } \\
\text { Coevaluación } \\
(\mathrm{N}=68) \\
\end{array}$} \\
\hline & & Antes & Después & Antes & Después \\
\hline $\begin{array}{l}\text { Sin conocimientos de } \\
\text { lo estudiado }\end{array}$ & $\begin{array}{l}\text { Con conocimientos de } \\
\text { lo estudiado }\end{array}$ & $2,59 \pm 1,2^{b}$ & $3,74 \pm 0,9$ & $2,43 \pm 1,2^{b}$ & $3,72 \pm 1,2$ \\
\hline Sin confianza & Con confianza & $3,05 \pm 1,0^{\mathrm{b}}$ & $3,54 \pm 0,9$ & $3,07 \pm 1,1^{\mathrm{b}}$ & $3,75 \pm 1,0$ \\
\hline $\begin{array}{l}\text { Menos flexible en mis } \\
\text { opiniones }\end{array}$ & $\begin{array}{l}\text { Más flexible en mis } \\
\text { opiniones }\end{array}$ & $2,99 \pm 0,8^{\mathrm{b}}$ & $3,49 \pm 0,8$ & $3,15 \pm 0,9^{\mathrm{b}}$ & $3,47 \pm 0,8$ \\
\hline Dependiente & Independiente & $3,17 \pm 1,1^{\mathrm{b}}$ & $3,43 \pm 1,0$ & $3,25 \pm 1,0^{\mathrm{b}}$ & $3,56 \pm 1,0$ \\
\hline Incompetente & Competente & $3,17 \pm 1,0^{\mathrm{b}}$ & $3,58 \pm 0,8$ & $3,16 \pm 1,0^{\mathrm{b}}$ & $3,84 \pm 0,9$ \\
\hline Nada entusiasmado & Entusiasmado & $2,89 \pm 1,0$ & $2,91 \pm 0,9^{\mathrm{a}}$ & $3,16 \pm 1,0$ & $3,37 \pm 1,1$ \\
\hline Menos creativo & Más creativo & $2,79 \pm 1,0^{\mathrm{b}}$ & $3,14 \pm 0,8^{\mathrm{a}}$ & $3,06 \pm 0,8^{\mathrm{b}}$ & $3,62 \pm 0,9$ \\
\hline $\begin{array}{l}a: p<0,01 \text { para la com } 1 \\
\text { b: } p<0,01 \text { para la com }\end{array}$ & $\begin{array}{l}\text { ación con-sin evaluación } \\
\text { ación antes-después }\end{array}$ & & & & \\
\hline
\end{tabular}

Tabla 2. Percepciones de los alumnos antes y después de realizar el trabajo en grupo

(Media \pm Desviación típica)

En la Tabla 3 se presentan las valoraciones sobre diferentes aspectos del funcionamiento del grupo así como sobre el conocimiento de los sujetos de sí mismos y del resto de los miembros del equipo. Los sujetos que participaron en equipos con coevaluación valoraron que el funcionamiento del trabajo en grupo fue mejor que los sujetos que participaron en equipos sin coevaluación $(\mathrm{z}=2,46 ; \mathrm{p}<0,01)$. Así mismo, los sujetos con coevaluación se mostraron más dispuestos a volver a trabajar con el mismo grupo $(\mathrm{z}=4,21 ; \mathrm{p}<0,01)$ y valoraron de manera más positiva el resultado $(\mathrm{z}=$ $1,92 ; \mathrm{p}<0,05)$ que aquellos que realizaron el trabajo en grupo sin coevaluación $(\mathrm{z}=$ $2,26 ; \mathrm{p}<0,05)$. Por el contrario, no hubo diferencias significativas en el grado de conocimiento adquirido por los sujetos sobre ellos mismos $(z=0,93 ; p>0,05)$, sin embargo los sujetos que participaron en equipos con coevaluación informaron haber aprendido mas del resto del grupo que aquellos que participaron en equipos sin coevaluación $(\mathrm{z}=2,26 ; \mathrm{p}<0,01)$. 


\begin{tabular}{lcc}
\hline & $\begin{array}{c}\text { Con } \\
\text { Coevaluación } \\
(\mathbf{N}=\mathbf{7 6})\end{array}$ & $\begin{array}{c}\text { Sin } \\
\text { Coevaluación } \\
(\mathbf{N = 6 8})\end{array}$ \\
\hline El trabajo en grupo ha funcionado (Muy mal=1 a Muy bien=5) & $3,97 \pm 1,0$ & $3,62 \pm 0,9^{* *}$ \\
\hline $\begin{array}{l}\text { Te gustaría volver a trabajar con el mismo grupo } \\
\text { (Nunca=1 a Seguro=4) }\end{array}$ & $3,54 \pm 0,5$ & $3,09 \pm 0,7^{* *}$ \\
\hline Valorarías el resultado (Muy mal=1 a Muy bien=5) & $3,96 \pm 1,0$ & $3,74 \pm 0,8^{*}$ \\
\hline Cuánto has aprendido de ti mismo (Muy poco=1 a Mucho=4) & $2,74 \pm 0,7$ & $2,64 \pm 0,6$ \\
\hline $\begin{array}{l}\text { Cuánto has aprendido del resto del grupo } \\
\text { (Muy poco=1 a Mucho=4) }\end{array}$ & $2,88 \pm 0,6$ & $2,62 \pm 0,7^{* *}$ \\
\hline$* \mathrm{p}<0,05 * * \mathrm{p}<0,01$ & & \\
\hline
\end{tabular}

Tabla 3. Percepciones de los alumnos sobre el funcionamiento del grupo

(Media \pm Desviación típica)

En la Tabla 4 se presentan las valoraciones sobre las competencias mejoradas y aquellas que aún deben mejorar en función de la introducción o no de coevaluación. La introducción o no de coevaluación en los grupos tuvo un efecto principal significativo sobre las competencias mejoradas $\left(\mathrm{F}_{8,135}=2,54 ; \mathrm{p}<0,05\right)$. Los contrastes univariados pusieron de manifiesto que los sujetos que participaron en equipos con coevaluación percibieron que su capacidad para trabajar con compañeros mejoró en mayor medida que aquellos que participaron en equipos sin coevaluación $\left(F_{1,142}=7,23 ; \mathrm{p}<0,01\right)$. No se observaron diferencias significativas en el grado en que los alumnos percibían haber mejorado en resolución de problemas $\left(\mathrm{F}_{1,142}<1\right)$, en su capacidad para investigar $\left(\mathrm{F}_{1,142}\right.$ $<1)$, para analizar datos $\left(\mathrm{F}_{1,142}=1,56 ; \mathrm{p}<0,05\right)$, presentar por escrito trabajos $\left(\mathrm{F}_{1,142}=\right.$ $1,54 ; \mathrm{p}>0,05)$, presentar oralmente trabajos $\left(\mathrm{F}_{1,142}=1,30 ; \mathrm{p}>0,05\right)$, planificar $\mathrm{y}$ organizar el trabajo $\left(\mathrm{F}_{1,142}=2,42 ; \mathrm{p}>0,05\right)$ y en organizar el tiempo $\left(\mathrm{F}_{1,142}<1\right)$. La introducción o no de coevaluación en los grupos no tuvo un efecto principal significativo sobre las competencias a mejorar $\left(\mathrm{F}_{8,135}=1,53 ; \mathrm{p}>0,05\right)$. 


\begin{tabular}{|c|c|c|c|c|}
\hline \multirow[b]{2}{*}{$\begin{array}{l}\text { Escala de } 1 \text { a } 5 \\
\text { (Nada a Mucho) }\end{array}$} & \multicolumn{2}{|c|}{ Aspectos Mejorados } & \multicolumn{2}{|c|}{ Aspectos a Mejorar } \\
\hline & $\begin{array}{c}\text { Con } \\
\text { Coevaluación } \\
(\mathrm{N}=76) \\
\end{array}$ & $\begin{array}{c}\text { Sin } \\
\text { Coevaluación } \\
(\mathrm{N}=68) \\
\end{array}$ & $\begin{array}{c}\text { Con } \\
\text { Coevaluación } \\
(\mathrm{N}=76) \\
\end{array}$ & $\begin{array}{c}\text { Sin } \\
\begin{array}{c}\text { Coevaluación } \\
(\mathrm{N}=68)\end{array} \\
\end{array}$ \\
\hline Resolución de problemas & $3,43 \pm 0,9$ & $3,41 \pm 0,8$ & $2,87 \pm 0,9$ & $3,01 \pm 1,0$ \\
\hline Investigar & $3,53 \pm 1,1$ & $3,67 \pm 0,9$ & $3,16 \pm 1,1$ & $2,93 \pm 1,0$ \\
\hline Analizar los datos & $3,56 \pm 0,9$ & $3,74 \pm 0,8$ & $3,19 \pm 1,0$ & $3,17 \pm 0,9$ \\
\hline $\begin{array}{l}\text { Presentación por escrito de } \\
\text { trabajos }\end{array}$ & $3,43 \pm 1,1$ & $3,22 \pm 0,9$ & $2,81 \pm 1,1$ & $2,82 \pm 1,1$ \\
\hline Presentación oral de trabajos & $3,01 \pm 1,3$ & $2,79 \pm 1,1$ & $2,99 \pm 1,3$ & $3,03 \pm 1,3$ \\
\hline Trabajar con compañeros & $3,66 \pm 0,8$ & $3,26 \pm 0,9^{* * *}$ & $2,63 \pm 1,0$ & $3,01 \pm 1,1$ \\
\hline Planificar y organizar el trabajo & $3,74 \pm 1,0$ & $3,49 \pm 0,9$ & $2,88 \pm 1,1$ & $3,18 \pm 1,1$ \\
\hline Organización del tiempo & $3,43 \pm 1,0$ & $3,39 \pm 1,1$ & $3,10 \pm 1,1$ & $3,17 \pm 1,1$ \\
\hline$* * \mathrm{p}<0,01$ & & & & \\
\hline
\end{tabular}

Tabla 4. Percepciones de los alumnos sobre las competencias mejoradas tras realizar el trabajo en grupo y las competencias a mejorar (Media \pm Desviación típica)

En la Tabla 5 se presentan las distribuciones de los sujetos en función de que considerasen mejorar o no ciertos aspectos si volvieran a realizar el trabajo en grupo y de la introducción o no de la coevaluación. No se observaron distribuciones diferentes en función de la introducción de la coevaluación en ninguno de los aspectos evaluados $\left(\chi^{2}(1)=0,20 ; p>0,05\right.$ para planificación; $\chi^{2}(1)=0,03 ; \mathrm{p}>0,05$ para trabajo de campo; $\chi^{2}(1)=1,11 ; p>0,05$ para organización del tiempo; $\chi^{2}(1)=0,08 ; p>0,05$ para investigación teórica; $\chi^{2}(1)=3,97 ; p>0,05$ para reparto de tareas; $\chi^{2}(1)=0,01 ; p>$ 0,05 para número de reuniones).

\begin{tabular}{lccc}
\hline & & $\begin{array}{c}\text { Con } \\
\text { Coevaluación } \\
(\mathbf{N = 7 6 )}\end{array}$ & $\begin{array}{c}\text { Sin } \\
\text { Coevaluación } \\
(\mathbf{N = 6 8 )}\end{array}$ \\
\hline Mayor planificación & $\mathbf{S i}$ & $61,9 \%$ & $58,1 \%$ \\
& $\mathbf{N o}$ & $38,1 \%$ & $41,9 \%$ \\
\hline Mayor trabajo de campo & $\mathbf{S i}$ & $30,2 \%$ & $31,7 \%$ \\
& $\mathbf{N o}$ & $69,8 \%$ & $68,3 \%$ \\
\hline Mejor organización del tiempo & $\mathbf{S i}$ & $62,5 \%$ & $53,5 \%$ \\
& $\mathbf{N o}$ & $37,5 \%$ & $46,5 \%$ \\
\hline Més investigación teórica & $\mathbf{S i}$ & $41,9 \%$ & $44,4 \%$ \\
& $\mathbf{N o}$ & $58,1 \%$ & $55,6 \%$ \\
\hline Mayor número de reuniones & $\mathbf{S i}$ & $41,3 \%$ & $58,6 \%$ \\
& $\mathbf{N o}$ & $58,7 \%$ & $41,4 \%$ \\
\hline
\end{tabular}

Tabla 5. Aspectos a mejorar para realizar el trabajo en grupo en un futuro 
Por último, la calificación sobre 10 puntos obtenida en el trabajo en los grupos en los que se introdujo la coevaluación $(6,69 \pm 1,69)$ no fue significativamente diferente a la obtenida en los grupos en los que no se introdujo $\left(6,41 \pm 1,70 ; \mathrm{t}_{142}=0,99 ; \mathrm{p}>0,05\right)$.

\section{Discusión}

El objetivo del presente estudio fue evaluar si las percepciones de los alumnos antes y después de la realización del trabajo, su percepción sobre diversos aspectos del funcionamiento del grupo y las competencias de trabajo adquiridas y las que consideran que deben mejorar, difería en función de que la contribución de los distintos miembros del grupo fuese o no coevaluada. Los resultados mostraron que los grupos en los se introdujo la coevaluación valoraron de forma más negativa la experiencia en general (menos agradable, inspiradora, positiva y creativa) que los grupos en los que no se introdujo la coevaluación. Sin embargo, los grupos con coevaluación percibieron que su competencia para trabajar en equipo había mejorado en mayor medida que los grupos sin coevaluación, valorando de forma más positiva tanto el funcionamiento como el resultado del trabajo e incrementaron en mayor medida sus conocimientos sobre el resto de los componentes.

Los grupos con coevaluación valoraron la experiencia como menos agradable, menos inspiradora, como una experiencia de aprendizaje menos positiva y menos creativa que aquellos que participaron en grupos sin coevaluación. Estudios previos han mostrado que para una minoría de estudiantes, la coevaluación presenta ciertos aspectos negativos. Algunos estudiantes se sienten incómodos o presionados al tener que evaluar a sus compañeros (White, 2009), tienen miedo a ser infra evaluados o a puntuar en exceso el trabajo de otros (Liu et al., 2001) o bien consideran que sus compañeros no tienen las habilidades necesarias para evaluarles y por tanto responden peor ante las críticas de sus compañeros (Wen y Tsai, 2006).

Cuando se solicitó a los alumnos que evaluasen los progresos que había supuesto la realización del trabajo en grupo, tanto los alumnos que participaron en grupos con coevaluación como aquellos que participaron en grupos sin coevaluación, tras la realización del trabajo se percibieron con más conocimientos de lo estudiado, con más confianza, más flexibles en sus opiniones, mas independientes, más competentes y más creativos. En cambio, cuando se les pidió que evaluasen en qué medida habían mejorado diferentes competencias gracias al trabajo realizado sólo observamos diferencias entre los grupos con y sin coevaluación en la capacidad para trabajar con compañeros, que mejoró en mayor medida en aquellos que participaron en grupos con coevaluación.

A pesar de no utilizar estrategias que contribuyan a mejorar la dinámica de grupo, el trabajo en grupo produce indudables beneficios. Tal y como señalan algunos teóricos de la educación (Johnson y Johnson, 1989; 1994; 2006) el trabajo en grupo genera soluciones más creativas ante problemas complejos, desarrolla la capacidad para solucionar problemas, incrementa el desarrollo cognitivo y la motivación de los estudiantes. El presente estudio pone de manifiesto que estos aspectos beneficiosos del trabajo en grupo son percibidos por los estudiantes. Así mismo, el hecho de que los 
grupos en los que se introdujo la coevaluación percibieran que su capacidad para trabajar en grupo mejoró en mayor medida que en los grupos donde no se introdujo la evaluación de los iguales, es congruente con lo informado en otros estudios (Hanrahan e Isaacs, 2001) y con la creencia de los profesores de que la coevaluación contribuye a facilitar y desarrollar las capacidades de interacción de los estudiantes (Karaca, 2009).

Los sujetos que participaron en grupos con coevaluación valoraron que el funcionamiento del trabajo fue mejor que los sujetos que participaron en grupos sin coevaluación. Por ello, los sujetos con coevaluación se mostraron más dispuestos a volver a trabajar con el mismo grupo y valoraron de manera más positiva el resultado que aquellos que realizaron el trabajo en grupo sin coevaluación. Por último, los sujetos que participaron en grupos con coevaluación informaron haber aprendido más del resto del grupo que, aquellos que participaron en equipos sin coevaluación. Parece que, a pesar de que los alumnos valoran la experiencia de trabajar en equipo de forma menos positiva, cuando se implementa la coevaluación, su percepción sobre el funcionamiento y el resultado del grupo es más positiva que cuando no se implementa la coevaluación y además, están en mayor medida dispuestos a repetir la experiencia con el mismo grupo. Estos datos, congruentes en parte con los aportados en otros estudios (Liu y Carless, 2006), parecen sugerir que la coevaluación sobre la contribución de los distintos miembros del equipo al trabajo puede ser una estrategia que ayude a estructurar el funcionamiento del grupo, mejorando el proceso y el producto del aprendizaje, aunque estos resultados no muestran efecto de la introducción de la coevaluación sobre las calificaciones obtenidas por los alumnos.

Este estudio presenta algunas limitaciones. La primera, es que el trabajo en grupo no se dotó de una estructura cooperativa, con lo que en dicho caso quizás los logros en relación a las competencias podrían haber sido mayores. Por otro lado, al ser alumnos de primero, la madurez de los mismos pudo haber influido, dado que los trabajos en grupo suponen un reto para los alumnos de primer año, mientras que en los alumnos de último curso, dicho aprendizaje se encuentra más avanzado (Garvin et al, 1995).. Así mismo el hecho de que el cuestionario se respondiera vía plataforma on-line de la asignatura, pudo influir de forma negativa en la baja tasa de respuesta. Por ello, para futuras experiencias sería más adecuado administrar el cuestionario en formato lápiz y papel en el aula de clase.

\section{Conclusiones}

En definitiva, a pesar de los beneficios que el trabajo en grupo supone para el aprendizaje, los docentes no pueden dejar de considerar los diversos aspectos negativos que surgen en la dinámica del grupo. Se hace necesario, por tanto, poner en marcha estrategias que faciliten el desarrollo de las habilidades de trabajo en grupo (Burdett, 2007). El uso de un sistema de coevaluación como el propuesto, ayuda a incrementar las habilidades para el trabajo conjunto, objetivo fundamental del trabajo en grupo (Asgari y Dall'Alba, 2011) y permite controlar la falta de contribución al trabajo por alguno de los miembros, uno de los problemas que más descontento genera con esta técnica. Finalmente, el hecho de que el alumno evalúe el proceso, no solo el resultado, 
es probable que le haya permitido un conocimiento más profundo del resto de los miembros y el realizar ajustes en la dinámica del grupo que desembocan en una mejor valoración tanto del funcionamiento del equipo como del resultado.

\section{Referencias bibliográficas}

ALÍAS A., GIL, C., RISCOS, A., VALCARCEL M., Y VICARIO E. (2006). Actas del Encuentro sobre la Formación del Profesorado Universitario. Universidad de Almería. 2006.

ASGARI, S., Y DALL'ALBA, G. (2011). Improving group functioning in solving realistic problems. International Journal for the Scholarship of Teaching and Learning, 5(1), 1-14.

BLANCO, A. (2009). Desarrollo y evaluación de competencias en educación superior. Madrid: Narcea.

BOURNER, H.; HUGHES, M., Y BOURNER T. (2001). First-year Undergraduate Experiences of Group Project Work. Assessment \& Evaluation in Higher Education, 26(1), 20-39.

BURDETT, J. (2007). Degrees of separation - balancing intervention and independence in group work assignments. The Australian Educational Researcher, 34(1), 55-71.

COLÁS, P. Y DE PABLOS, J. (2005). La Universidad en la Unión Europea. El Espacio Europeo de Educación Superior y su impacto en la docencia. Málaga: Aljibe.

CREBERT, G., BATES, M., BELL, B., PATRICK, C. J., Y CRAGNOLINI, V. (2004). Developing generic skills at university, during work placement and in employment: graduates' perceptions. Higher Education Research \& Development, 23(2), 147-165.

DRAPER, S.W. (2007). A momentary review of assessment principles. REAP Conference - Assessment design for learner responsibility, 9-31 May. Recuperado de http://reap.ac.uk.

FALCHICOV, N. (1988). Self and peer assessment of a group project designed to promote the skills of capability. Programmed Learning and Educational Technology, 25(4), 327-339.

FURNHAM, A. (1997). The Psychology of Behavior at Work. The Individual in the Organization. London: Psychology Press.

GÁMEZ, M. J., Y TORRES, C. (2013). Las técnicas de grupo como estrategia metodológica en la adquisición de la competencia de trabajo en equipo de los alumnos universitarios. Journal for Educators, Teachers and Trainers, 4(1), 14-25.

GARVIN, J., BUTCHER, A., STEFANI, A., TARIQ, V., LEWIS, N., BLUMSOM, R., GOVIER, R., Y HILL, J. (1995). Group projects for 1st-year university students: an evaluation. Assessment \& Evaluation in Higher Education, 20, 279-294.

HANRAHAN, S.J. Y ISAACS, G. (2001). Assessing self- and peer-assessment: the student's views. Higher Education Research \& Development, 20(1), 53-70.

JOHNSON, D. W., Y JOHNSON, R.T. (1989). Cooperation and Competitions. Theory and Research. Edina, MN. Interaction Book Company. 
JOHNSON, D. W. Y JOHNSON, R. T. (1994). Learning together and alone: Cooperative, competitive, and individualistic learning ( $4^{\mathrm{a}}$ Ed.). Needham Heights, M.A: Allyn and Bacon

JOHNSON, D. W. Y JOHNSON, R.T. (2006). Joining together: group theory and group skills $\left(9^{\mathrm{a}}\right.$ Ed.). Boston: Pearson.

JOHNSTON L. Y MILES, L. (2004). Assessing contributions to group assignments. Assessment \& Evaluation in Higher Education, 29(6), 751-767.

KARACA, E. (2009). An Evaluation of Teacher Trainees' Opinions of the Peer Assessment in Terms of Some Variables. World Applied Sciences Journal, 6(1), 123-128.

KAUFMAN, D. B.; FELDER, R. M., Y FULlER, H. (2000). Accounting for individual effort in cooperative learning teams. Journal of Engineering Education, 89(2), 133-140.

LEJK, M., Y WYVILL, M. (2001). Peer assessment of contributions to a Group Project: a comparison of holistic and categorybased approaches, Assessment \& Evaluation in Higher Education, 26(1), 61-72.

LIU, E.; LIN, S.; CHIU, C., Y YUAN, S. (2001). Web-based peer review: the learner as both adapter and reviewer, IEEE Transactions on Education, 44(3), 246-251.

LIU, N.F. Y CARLESS, D. (2006). Peer feedback: the learning element of peer assessment. Teaching in Higher Education, 11(3), 279-290.

LIVINGSTONE, D. Y LYNCH, K. (2002). Group projet work and student-centred active learning: Two different experiences. Journal of Geography in Higher Education, 20(2), 217-237.

LÓPEZ, F. (2005). Metodología participativa en la Enseñanza Universitaria. Madrid: Narcea.

MCCONNELL, D. (2005). Examining the dynamics of networked e-learning groups and communities. Studies in Higher Education, 30(1), 25-42.

MORALES, P. (2008). Estrategias para evaluar y calificar el producto del equipo. En Prieto, Leonor (coord.). La Enseñanza como entrada en el aprendizaje: estrategias útiles para el profesorado (pp 151-169). Barcelona: Octaedro.

MORENO, S.; BAJO, M. T., MOYA, M., MALDONADO, A., Y TUDELA, P. (2007). Las competencias en el nuevo paradigma educativo para Europa. Vicerrectorado de Planificación, Calidad y Evaluación Docente, Universidad de Granada.

SANTOS-REGO, M. A., LORENZO-MOLEDO, M. M., Y PRIEGUE-CAAMAÑO, D. (2009). Aprendizaje cooperativo: práctica pedagógica para el desarrollo escolar y cultural. magis. Revista Internacional de Investigación Educativa, 2, 289-303.

SCAGNOLI, N. (2005). Estrategias para motivar el aprendizaje colaborativo en cursos a distancia. En Illinois Research and Scholarship. Recuperado de http://hdl.handle.net/2142/10681

SLAVIN, R.E. (1987). Cooperative Learning: Student Teams (2 ${ }^{a}$ Ed.). Wahington: DC:National Education Association.

VALLEJO, M., Y MOLINA, J. (2011). Análisis de las metodologías activas en el grado de maestro en educación infantil: la perspectiva del alumnado. REIFOP, 14(1), 207-217. 
VILCHES, A. Y GIL-PÉREZ, D. (2011). El trabajo cooperativo en las clases de ciencias: una estrategia imprescindible pero aún infrautilizada. Alambique. Didáctica de las Ciencias Experimentales, 69, 73-79

WEN, M.L. Y TSAI, C.C. (2006). University student's percepcions of and attitudes toward (online) peer assessment. Higher Education, 51, 27-44.

WHITE, E. (2009). Student perspectives of peer assessment for learning in a public speaking course. Asian EFL Journal, 33(1), 1-36.

YAZICI, H. J. (2005). A study of collaborative learning style and team learning performance. Education + training, 47(3), 216-229.

\section{Correspondencia con autores}

Maribel BARRIOPEDRO MORO

C/ Martín Fierro, 7

Ciudad Universitaria

28040 Madrid

e-mail: misabel.barriopedro@upm.es

Cristina LÓPEZ DE SUBIJANA

C/ Martín Fierro, 7

Ciudad Universitaria

28040 Madrid

e-mail: c.lopezdesubijana@upm.es

Miguel Ángel GÓMEZ RUANO

C/ Martín Fierro, 7

Ciudad Universitaria

28040 Madrid

e-mail: miguelangel.gomez.ruano@upm.es

Antonio RIVERO HERRAIZ

C/ Martín Fierro, 7

Ciudad Universitaria

28040 Madrid

e-mail: antonio.rivero@upm.es 